Kragujevac Journal of Mathematics

Volume 45(3) (2021), Pages 439-448.

\title{
APPLICATION OF JACOBI POLYNOMIAL AND MULTIVARIABLE ALEPH-FUNCTION IN HEAT CONDUCTION IN NON-HOMOGENEOUS MOVING RECTANGULAR PARALLELEPIPED
}

\author{
DINESH KUMAR ${ }^{1}$ AND FRÉDÉRIC AYANT ${ }^{2,3}$
}

\begin{abstract}
The present paper deals with an application of Jacobi polynomial and multivariable Aleph-function to solve the differential equation of heat conduction in non-homogeneous moving rectangular parallelepiped. The temperature distribution in the parallelepiped, moving in a direction of the length ( $x$-axis) between the limits $x=-1$ and $x=1$ has been considered. The conductivity and the velocity have been assumed to be variables. We shall see two particular cases and the cases concerning Aleph-function of two variables and the $I$-function of two variables.
\end{abstract}

\section{Introduction AND PRELIMINARIES}

We suppose the parallelepiped has heat conductivity $K$, density $\rho$, diffusivity $k$ and specific heat $\sigma$. The partial differential equation satisfied by the temperature $v(x, y, z, t)$ at any time $t$ in a homogeneous parallelepiped bounded by the planes $y=0$ and $y=b, z=0$ and $z=c$, moves with a constant velocity $U$ in the direction of its length ( $x$-axis) between the limits $x=-1$ and $x=1$, on the lines of Carslaw and Jaeger [4, page 155, (1)] is

$$
k\left[\frac{\partial^{2} v}{\partial x^{2}}+\frac{\partial^{2} v}{\partial y^{2}}+\frac{\partial^{2} v}{\partial z^{2}}\right]-U \frac{\partial v}{\partial x}-\frac{\partial v}{\partial t}=0
$$

where $k=\frac{K}{\rho \sigma}$. If we consider a non-homogeneous parallelepiped of variable conductivity $k^{\prime}\left(1-x^{2}\right)$ and the velocity $k_{0}[(\alpha-\beta)+(\alpha+\beta) x]$, where $k^{\prime}, k_{0}, \alpha$ and $\beta$ are

Key words and phrases. Jacobi polynomial, heat conduction, Aleph-function of several variables, aleph-function of two variables, $I$-function of two variables.

2010 Mathematics Subject Classification. Primary: 33C99, 33C60. Secondary: 44A20.

DOI 10.46793/KgJMat2103.439K

Received: April 02, 2018.

Accepted: February 15, 2019. 
constants, the partial differential equation (1.1) reduces to

$$
\frac{\partial v}{\partial t}=k_{0}\left[\left(1-x^{2}\right) \frac{\partial^{2} v}{\partial x^{2}}+((\beta-\alpha)-(\alpha+\beta+2) x) \frac{\partial v}{\partial x}\right]+k\left[\frac{\partial^{2} v}{\partial y^{2}}+\frac{\partial^{2} v}{\partial z^{2}}\right]=0
$$

where $k_{0}=\frac{k^{\prime}}{\rho \sigma}, \operatorname{Re}(\alpha)>-1, \operatorname{Re}(\beta)>-1$.

As physical example we can consider the temperature distribution of the moving mercury parallelepiped between the planes $x=-1, x=1, y=0$ and $y=b, z=0$ and $z=c$ connected by two reservoirs of the mercury at the two ends. The variable flow in the mercury at the end $x=-1$ with a certain speed. The initial temperature distribution in the parallelepiped of mercury can be taken to be $f(x, y, z)$. The surfaces $y=0$ and $y=b, z=0$ and $z=c$ of the parallelepiped are supposed to be insulated. The ends $x=-1$ and $x=1$ of the mercury parallelepiped should also be insulated as the conductivity vanishes there.

\section{Solution of the Problem}

By assuming the solution of the partial differential equation (1.2) as $v(x, y, z, t)=$ $X(x) Y(y) Z(z) T(t)$, the solution of the partial differential equation (1.2) reduces to

$$
\frac{1}{T} \frac{d T}{d t}=\frac{k_{0}}{X}\left[\left(1-x^{2}\right) \frac{d^{2} X}{d x^{2}}+(-\alpha+\beta-(\alpha+\beta+2) x) \frac{d X}{d x}\right]+\frac{k}{Y} \frac{d^{2} Y}{d y^{2}}+\frac{k}{Z} \frac{d^{2} Z}{d z^{2}} .
$$

Now, taking

$$
\begin{aligned}
\frac{k_{0}}{X}\left[\left(1-x^{2}\right) \frac{d^{2} X}{d x^{2}}+(-\alpha+\beta-(\alpha+\beta+2) x) \frac{d X}{d x}\right] & =-k_{0} n(n+\alpha+\beta+1), \\
\frac{k}{Y} \frac{d^{2} Y}{d y^{2}} & =-k \lambda^{2},
\end{aligned}
$$

and $\frac{k}{Z} \frac{d^{2} Z}{d z^{2}}=-k v^{2}, \lambda, v$ being constants, $n$ being positive integer, we obtain the following equations

$$
\begin{gathered}
\left(1-x^{2}\right) \frac{d^{2} X}{d x^{2}}+(-\alpha+\beta-(\alpha+\beta+2) x) \frac{d X}{d x}+n(n+\alpha+\beta+1) X=0, \\
\frac{d^{2} Y}{d y^{2}}+\lambda^{2} y=0, \\
\frac{d^{2} Z}{d z^{2}}+v^{2} z=0,
\end{gathered}
$$

and

$$
\frac{d T}{d t}=\left[-n(n+\alpha+\beta+1)-k\left(\lambda^{2}+v^{2}\right)\right] T .
$$

The (2.1) is the differential equation of Jacobi polynomials and its solution is

$$
X=P_{n}^{(\alpha, \beta)}(x) .
$$


The solution of (2.2), (2.3) and (2.4) are

$$
\begin{aligned}
& Y=A \cos \lambda y+B \sin \lambda y \\
& Z=C \cos v z+D \sin v z
\end{aligned}
$$

and

$$
T=E \exp \left[-\left(k_{0} n(n+\alpha+\beta+1)+k\left(\lambda^{2}+v^{2}\right)\right) t\right],
$$

where $A, B, C, D, E$ are constants.

Hence, the general solution of (1.2), the temperature distribution at any point $M(x, y, z)$ of the parallelepiped at time $t$ is given by

$$
\begin{aligned}
v(x, y, z, t)= & \exp \left[-\left(k_{0} n(n+\alpha+\beta+1)+k\left(\lambda^{2}+v^{2}\right)\right) t\right] P_{n}^{(\alpha, \beta)}(x) \\
& \times[A \cos \lambda y+B \sin \lambda y][C \cos v z+D \sin v z],
\end{aligned}
$$

if no heats flows from the surfaces $y=0$ and $y=b, z=0$ and $z=c,\left(\frac{\partial v}{\partial y}\right)_{y=b=0}=0$, $\left(\frac{\partial v}{\partial z}\right)_{z=c=0}=0$ for all $x$ and $t$. These demand $B=0, D=0, \lambda=\frac{m \pi}{b}, v=\frac{l \pi}{c}$, where $m, l=0,1,2, \ldots$.

Therefore, the solution $(2.5)$ reduces to

$$
\begin{aligned}
v(x, y, z, t)= & \sum_{n, m, l=0}^{\infty} A_{n m l} \exp \left[-\left(k_{0} n(n+\alpha+\beta+1)+k\left(\lambda^{2}+v^{2}\right)\right) t\right] \\
& \times P_{n}^{(\alpha, \beta)}(x) \cos \frac{m \pi}{b} y \cos \frac{l \pi}{c} z .
\end{aligned}
$$

Here, $\operatorname{Re}(\alpha)>-1, \operatorname{Re}(\beta)>-1$ and $A_{n m l}$ are constants. If the initial temperature distribution in parallelepiped is given by

$$
\sum_{n, m, l=0}^{\infty} A_{n m l} P_{n}^{(\alpha, \beta)}(x) \cos \frac{m \pi}{b} y \cos \frac{l \pi}{c} z .
$$

Now, multiplying both sides of $(2.6)$ by $(1-x)^{\alpha}(1+x)^{\beta} P_{n}^{(\alpha, \beta)}(x) \cos \frac{m \pi}{b} y \cos \frac{l \pi}{c} z$, integrating both sides between $x=-1$ and $x=1, y=0$ and $y=b, z=0$ and $z=c$, and applying the result [5, Vol. II. Page 285, (5)]

$$
\int_{-1}^{1}(1-x)^{\alpha}(1+x)^{\beta}\left[P_{n}^{(\alpha, \beta)}(x)\right]^{2} \mathrm{~d} x=\frac{2^{\alpha+\beta+1} \Gamma(\alpha+n+1) \Gamma(\beta+n+1)}{n !(\alpha+\beta+2 n+1) \Gamma(\alpha+\beta+n+1)},
$$

with $\operatorname{Re}(\alpha)>-1, \operatorname{Re}(\beta)>-1$, we obtain

$$
\begin{aligned}
A_{m n l}= & \frac{n !(\alpha+\beta+2 n+1) \Gamma(\alpha+\beta+n+1)}{2^{\alpha+\beta+1} \Gamma(\alpha+n+1) \Gamma(\beta+n+1)} \\
& \times \int_{0}^{c} \int_{0}^{b} \int_{-1}^{1}(1-x)^{\alpha}(1+x)^{\beta} P_{n}^{(\alpha, \beta)}(x) \cos \frac{m \pi}{b} y \cos \frac{l \pi}{c} z f(x, y, z) d x d y d z .
\end{aligned}
$$


Hence, the temperature distribution in the non-homogeneous moving rectangular parallelepiped is given by

$$
\begin{aligned}
& v(x, y, z, t) \\
= & \frac{1}{2^{\alpha+\beta-1} b c} \sum_{n, m, l=0}^{\infty} \frac{n !(\alpha+\beta+2 n+1) \Gamma(\alpha+\beta+n+1)}{2^{\alpha+\beta+1} \Gamma(\alpha+n+1) \Gamma(\beta+n+1)} \\
& \times \exp \left[-\left(k_{0} n(n+\alpha+\beta+1)+k \pi^{2}\left(\frac{m^{2}}{b^{2}}+\frac{l^{2}}{c^{2}}\right)\right) t\right] P_{n}^{(\alpha, \beta)}(x) \cos \frac{m \pi}{b} y \cos \frac{l \pi}{c} z \\
& \times \int_{0}^{c} \int_{0}^{b} \int_{-1}^{1}(1-x)^{\alpha}(1+x)^{\beta} P_{n}^{(\alpha, \beta)}(x) \cos \frac{m \pi}{b} y \cos \frac{l \pi}{c} z f(x, y, z) d x d y d z,
\end{aligned}
$$

with $\operatorname{Re}(\alpha)>-1, \operatorname{Re}(\beta)>-1$.

Remark 2.1. Prasad and Maurya [10] have given application of Jacobi polynomial and multivariable $H$-function in heat conduction in non-homogeneous moving rectangular parallelepiped; Simões et al. [9] have studied Green's functions for heat conduction for unbounded and bounded rectangular spaces.

\section{Multivariable Aleph-Function}

For an illustration, if we take $f(x, y, z)=f_{1}(x) f_{2}(y) f_{3}(z), f_{2}(y)=e^{-\mu y}, f_{3}(z)=$ $e^{-\delta z}$ and $f_{1}(x)$ to be the most general special function in the form of multivariable Aleph-function.

The multivariable Aleph-function is a generalization of the multivariable $H$-function defined by Srivastava and Panda [14,15]. The multivariable Aleph-function is defined by means of the multiple contour integral $[3,7]$ :

$$
\begin{aligned}
& \aleph\left(z_{1}, \ldots, z_{r}\right) \\
& =\aleph_{p_{i}, q_{i}, \tau_{i} ; R: p_{i}(1), q_{i}(1), \tau_{i}(1) ; R^{(1)} ; \ldots ; p_{i}(r), q_{i}(r) ; \tau_{i}(r) ; R^{(r)}}^{0, \mathfrak{n}: m_{1}, n_{1}, \ldots, m_{r}, n_{r}}\left(\begin{array}{l|l}
z_{1} & {\left[\left(a_{j} ; \alpha_{j}^{(1)}, \ldots, \alpha_{j}^{(r)}\right)_{1, \mathfrak{n}}\right.}
\end{array}\right], \\
& \begin{array}{l}
{\left[\tau_{i}\left(a_{j i} ; \alpha_{j i}^{(1)}, \ldots, \alpha_{j i}^{(r)}\right)_{\mathfrak{n}+1, p_{i}}\right]:\left[\left(c_{j}^{(1)}\right),\left(\gamma_{j}^{(1)}\right)_{1, n_{1}}\right],\left[\tau_{i^{(1)}}\left(c_{j i(1)}^{(1)}, \gamma_{j i^{(1)}}^{(1)}\right)_{n_{1}+1, p_{i}^{(1)}}\right]} \\
{\left[\tau_{i}\left(b_{j i} ; \beta_{j i}^{(1)}, \cdots, \beta_{j i}^{(r)}\right)_{m+1, q_{i}}\right]:\left[\left(d_{j}^{(1)}\right),\left(\delta_{j}^{(1)}\right)_{1, m_{1}}\right],\left[\tau_{i^{(1)}}\left(d_{j i^{(1)}}^{(1)}, \delta_{j i^{(1)}}^{(1)}\right)_{m_{1}+1, q_{i}^{(1)}}\right] ;}
\end{array} \\
& \begin{array}{l}
\ldots ;\left[\left(c_{j}^{(r)}\right),\left(\gamma_{j}^{(r)}\right)_{1, n_{r}}\right],\left[\tau_{i^{(r)}}\left(c_{j i(r)}^{(r)}, \gamma_{j i(r)}^{(r)}\right)_{n_{r}+1, p_{i}^{(r)}}\right] \\
\ldots ;\left[\left(d_{j}^{(r)}\right),\left(\delta_{j}^{(r)}\right)_{1, m_{r}}\right],\left[\tau_{i(r)}\left(d_{j i(r)}^{(r)}, \delta_{j i(r)}^{(r)}\right)_{m_{r}+1, q_{i}^{(r)}}\right]
\end{array}
\end{aligned}
$$

$$
=\frac{1}{(2 \pi \omega)^{r}} \int_{L_{1}} \cdots \int_{L_{r}} \psi\left(s_{1}, \ldots, s_{r}\right) \prod_{k=1}^{r} \theta_{k}\left(s_{k}\right) z_{k}^{s_{k}} d s_{1} \cdots d s_{r},
$$


with $\omega=\sqrt{-1}$

$$
\psi\left(s_{1}, \ldots, s_{r}\right)=\frac{\prod_{j=1}^{\mathfrak{n}} \Gamma\left(1-a_{j}+\sum_{k=1}^{r} \alpha_{j}^{(k)} s_{k}\right)}{\sum_{i=1}^{R}\left[\tau_{i} \prod_{j=\mathfrak{n}+1}^{p_{i}} \Gamma\left(a_{j i}-\sum_{k=1}^{r} \alpha_{j i}^{(k)} s_{k}\right) \prod_{j=1}^{q_{i}} \Gamma\left(1-b_{j i}+\sum_{k=1}^{r} \beta_{j i}^{(k)} s_{k}\right)\right]}
$$

and

$$
\theta_{k}\left(s_{k}\right)=\frac{\prod_{j=1}^{m_{k}} \Gamma\left(d_{j}^{(k)}-\delta_{j}^{(k)} s_{k}\right) \prod_{j=1}^{n_{k}} \Gamma\left(1-c_{j}^{(k)}+\gamma_{j}^{(k)} s_{k}\right)}{\sum_{i^{(k)}=1}^{R^{(k)}}\left[\tau_{i^{(k)}} \prod_{j=m_{k}+1}^{q_{i(k)}} \Gamma\left(1-d_{j i^{(k)}}^{(k)}+\delta_{j i^{(k)}}^{(k)} s_{k}\right) \prod_{j=n_{k}+1}^{p_{i(k)}} \Gamma\left(c_{j i(k)}^{(k)}-\gamma_{j i^{(k)}}^{(k)} s_{k}\right)\right]} .
$$

For more details, see Ayant [1]. The condition for absolute convergence of multiple Mellin-Barnes type contour can be obtained by extension of the corresponding conditions for multivariable $H$-function given by

$$
\left|\arg z_{k}\right|<\frac{1}{2} A_{i}^{(k)} \pi
$$

where

$$
\begin{aligned}
A_{i}^{(k)}= & \sum_{j=1}^{\mathfrak{n}} \alpha_{j}^{(k)}-\tau_{i} \sum_{j=\mathfrak{n}+1}^{p_{i}} \alpha_{j i}^{(k)}-\tau_{i} \sum_{j=1}^{q_{i}} \beta_{j i}^{(k)}+\sum_{j=1}^{n_{k}} \gamma_{j}^{(k)}-\tau_{i}(k) \sum_{j=n_{k}+1}^{p_{i}(k)} \gamma_{j i}^{(k)} \\
& +\sum_{j=1}^{m_{k}} \delta_{j}^{(k)}-\tau_{i(k)} \sum_{j=m_{k}+1}^{q_{i^{(k)}}} \delta_{j i^{(k)}}^{(k)}>0,
\end{aligned}
$$

with $k=1, \ldots, r, i=1, \ldots, R, i^{(k)}=1, \ldots, R^{(k)}$.

The complex numbers $z_{i}$ are not zero. Throughout this document, we assume the existence and absolute convergence conditions of the multivariable Aleph-function. For convenience, we shall use the following notations in this paper.

$$
\begin{aligned}
V= & m_{1}, n_{1} ; \ldots ; m_{r}, n_{r} \\
W= & p_{i^{(1)}}, q_{i(1)}, \tau_{i^{(1)}} ; R^{(1)} ; \ldots ; p_{i(r)}, q_{i(r)}, \tau_{i^{(r)}} ; R^{(r)}, \\
A= & \left\{\left(a_{j} ; \alpha_{j}^{(1)}, \ldots, \alpha_{j}^{(r)}\right)_{1, n}\right\},\left\{\tau_{i}\left(a_{j i} ; \alpha_{j i}^{(1)}, \ldots, \alpha_{j i}^{(r)}\right)_{n+1, p_{i}}\right\}:\left\{\left(c_{j}^{(1)} ; \gamma_{j}^{(1)}\right)_{1, n_{1}}\right\}, \\
& \left\{\tau_{i^{(1)}}\left(c_{j i(1)}^{(1)} ; \gamma_{j i^{(1)}}^{(1)}\right)_{n_{1}+1, p_{i}(1)}\right\} ; \ldots ;\left\{\left(c_{j}^{(r)} ; \gamma_{j}^{(r)}\right)_{1, n_{r}}\right\},\left\{\tau_{i^{(r)}}\left(c_{j i^{(r)}}^{(r)} ; \gamma_{j i^{(r)}}^{(r)}\right)_{n_{r}+1, p_{i}(r)}\right\}, \\
B= & \left\{\tau_{i}\left(b_{j i} ; \beta_{j i}^{(1)}, \ldots, \beta_{j i}^{(r)}\right)_{m+1, q_{i}}\right\}:\left\{\left(d_{j}^{(1)} ; \delta_{j}^{(1)}\right)_{1, m_{1}}\right\},\left\{\tau_{i^{(1)}}\left(d_{j i^{(1)}}^{(1)} ; \delta_{j i^{(1)}}^{(1)}\right)_{m_{1}+1, q_{i}(1)}\right\} ; \\
& \ldots ;\left\{\left(d_{j}^{(r)} ; \delta_{j}^{(r)}\right)_{1, m_{r}}\right\},\left\{\tau_{i_{(r)}}\left(d_{j i(r)}^{(r)} ; \delta_{j i(r)}^{(r)}\right)_{m_{r}+1, q_{i}(r)}\right\} .
\end{aligned}
$$

Let

$$
f_{1}(x)=\aleph_{p_{i}, q_{i}, \tau_{i} ; R: W}^{0, \mathbf{n}: V}\left(\begin{array}{c|c}
p_{1}(1-x)^{m_{1}^{\prime}}(1+x)^{m_{1}^{\prime \prime}} & A \\
\vdots & \vdots \\
p_{r}(1-x)^{m_{r}^{\prime}}(1+x)^{m_{r}^{\prime \prime}} & B
\end{array}\right)
$$


Substituting for $f_{1}(x), f_{2}(y)$ and $f_{3}(z)$ in equation (2.7), which is justifiable under the given conditions, we evaluate the $y$ and $z$-integrals, first and the write the multivariable Aleph-function into the Mellin-Barnes contour integral with the help of (3.1), apply the result [5, Vol. II, page 284, (3)],

$$
\begin{aligned}
& \int_{-1}^{1}(1-x)^{l}(1+x)^{\sigma} P_{n}^{(\alpha, \beta)}(x) d x \\
= & \frac{2^{l+\sigma+1} \Gamma(l+1) \Gamma(\sigma+1)}{\Gamma(l+\sigma+2)} \times{ }_{3} F_{2}(-n, \alpha+\beta+n+1, l+1 ; \alpha+1, l+\sigma+2 ; 1),
\end{aligned}
$$

with $\operatorname{Re}(\alpha)>-1, \operatorname{Re}(\beta)>-1$, and finally interpret the resulting $\Gamma$-functions with the definition of multivariable Aleph-function. The temperature distribution in a non-homogeneous moving rectangular parallelepiped is then

$$
\begin{aligned}
& v(x, y, z, t)=\frac{\mu \delta e^{-(\mu b+\delta c)} \Gamma(\alpha+1)}{b c 2^{\alpha+\beta-1}} \sum_{n, m, l, N=0}^{\infty} \frac{\left(1-(-)^{m}\right)\left(1-(-)^{l}\right)}{\left(\mu^{2}+m^{2} \pi^{2} / b^{2}\right)\left(\delta^{2}+l^{2} \pi^{2} / c^{2}\right)} \\
& \times \frac{n !(\alpha+\beta+2 n+1) \Gamma(\alpha+\beta+n+N+1) \Gamma(-n+N)}{N ! \Gamma(\alpha+N+1) \Gamma(\alpha+n+1) \Gamma(\beta+n+1) \Gamma(-n)} \\
& \times \exp \left[-\left\{k_{0} n(n+\alpha+\beta+1)+k \pi^{2}\left(\frac{m^{2}}{b^{2}}+\frac{l^{2}}{c^{2}}\right)\right\} t\right] \\
& \times P_{n}^{(\alpha, \beta)}(x) \cos \frac{m \pi}{b} y \cos \frac{l \pi}{c} z \aleph_{p_{i}+2, q_{i}+1, \tau_{i} ; R: W}^{0, \mathbf{n}+2: V}\left(\begin{array}{c}
p_{1} 2^{m_{1}^{\prime}+m_{1}^{\prime \prime}} \\
\vdots \\
p_{r} 2^{m_{r}^{\prime}+m_{r}^{\prime \prime}}
\end{array}\right) \\
& \left.\begin{array}{c}
\left(-\alpha-N: m_{1}^{\prime}, \ldots, m_{r}^{\prime}\right),\left(-\beta: m_{1}^{\prime \prime}, \ldots, m_{r}^{\prime \prime}\right), A \\
\vdots \\
\left(-\alpha-\beta-N-1: m_{1}^{\prime}+m_{1}^{\prime \prime}, \ldots, m_{r}^{\prime}+m_{r}^{\prime \prime}\right), B
\end{array}\right) .
\end{aligned}
$$

Provide that $\operatorname{Re}(\alpha)>-1, \operatorname{Re}(\beta)>-1, m_{i}^{\prime}, m_{i}^{\prime \prime}>0$ for $i=1, \ldots, r$, and

$$
\begin{aligned}
& \operatorname{Re}(\alpha+1)+\sum_{i=1}^{r} m_{i}^{\prime} \min _{1 \leqslant j \leqslant m_{i}} \operatorname{Re}\left[\left(\frac{d_{j}^{(i)}}{\delta_{j}^{(i)}}\right)\right]>0, \\
& \operatorname{Re}(\beta+1)+\sum_{i=1}^{r} m_{i}^{\prime \prime} \min _{1 \leqslant j \leqslant m_{i}} \operatorname{Re}\left[\left(\frac{d_{j}^{(i)}}{\delta_{j}^{(i)}}\right)\right]>0,
\end{aligned}
$$

$\left|\arg p_{k}\right|<\frac{1}{2} A_{i}^{(k)} \pi$, where $A_{i}^{(k)}$ is defined by (3.2).

Remark 3.1. For detail and applications of Aleph-function, the reader can refer recent work $[2,8,16]$. 


\section{Particular Cases}

(a) When the rectangular parallelepiped moves with uniform velocity, the partial differential equation (1.2) reduces to the unsteady case of the partial differential equation (1) of Carslaw and Jaeger [4] with no radiation but with variable conductibility. We have $\alpha+\beta=0$ and

$$
\frac{\partial v}{\partial t}=k_{0}\left(\left(1-x^{2}\right) \frac{\partial^{2} v}{\partial x^{2}}+(\beta-\alpha-2 x) \frac{\partial v}{\partial x}\right)+k\left(\frac{\partial^{2} v}{\partial y^{2}}+\frac{\partial^{2} v}{\partial z^{2}}\right)=0,
$$

and the temperature distribution in the parallelepiped between $x=-1$ and $x=$ $1, y=0$ and $y=b, z=0$ and $z=c$ is given by

$$
\begin{aligned}
& v(x, y, z, t) \\
& =\frac{2 \mu \delta e^{-(\mu b+\delta c)} \Gamma(\alpha+1)}{b c} \sum_{n, m, l, N=0}^{\infty} \frac{\left(1-(-)^{m}\right)\left(1-(-)^{l}\right)}{\left(\mu^{2}+m^{2} \pi^{2} / b^{2}\right)\left(\delta^{2}+l^{2} \pi^{2} / c^{2}\right)} \\
& \times \frac{n !(2 n+1) \Gamma(n+N+1) \Gamma(-n+N)}{N ! \Gamma(\alpha+N+1) \Gamma(\alpha+n+1) \Gamma(\beta+n+1) \Gamma(-n)} \\
& \times \exp \left[-\left(k_{0} n(n+1)+k \pi^{2}\left(\frac{m^{2}}{b^{2}}+\frac{l^{2}}{c^{2}}\right)\right) t\right] P_{n}^{(\alpha, \beta)}(x) \cos \frac{m \pi}{b} y \cos \frac{l \pi}{c} z \\
& \times \aleph_{p_{i}+2, q_{i}+1, \tau_{i} ; R: W}^{0, \mathbf{n}+2:}\left(\begin{array}{c|c}
p_{1} 2^{m_{1}^{\prime}+m_{1}^{\prime \prime}} & \left(-\alpha-N: m_{1}^{\prime}, \ldots, m_{r}^{\prime}\right),\left(-\beta: m_{1}^{\prime \prime}, \ldots, m_{r}^{\prime \prime}\right), A \\
\vdots & \vdots \\
p_{r} 2^{m_{r}^{\prime}+m_{r}^{\prime \prime}} & \left(-N-1: m_{1}^{\prime}+m_{1}^{\prime \prime}, \ldots, m_{r}^{\prime}+m_{r}^{\prime \prime}\right), B
\end{array}\right),
\end{aligned}
$$

under the same condition that (3.4) with $\alpha+\beta=0$.

(b) When the parallelepiped is stationary between $x=-1$ and $x=1$, we have $\alpha=\beta=0$ and the partial differential equation (1.2) reduces to

$$
\frac{\partial v}{\partial t}=k_{0}\left(\left(1-x^{2}\right) \frac{\partial^{2} v}{\partial x^{2}}-2 x \frac{\partial v}{\partial x}\right)+k\left(\frac{\partial^{2} v}{\partial y^{2}}+\frac{\partial^{2} v}{\partial z^{2}}\right)=0,
$$

and the temperature distribution in the parallelepiped between $x=-1$ and $x=$ $1, y=0$ and $y=b, z=0$ and $z=c$ is given by

$$
\begin{aligned}
& v(x, y, z, t) \\
= & \frac{2 \mu \delta e^{-(\mu b+\delta c)}}{b c} \sum_{n, m, l, N=0}^{\infty} \frac{\left(1-(-)^{m}\right)\left(1-(-)^{l}\right)}{\left(\mu^{2}+m^{2} \pi^{2} / b^{2}\right)\left(\delta^{2}+l^{2} \pi^{2} / c^{2}\right)} \\
\times & \frac{n !(2 n+1) \Gamma(n+N+1) \Gamma(-n+N)}{N ! \Gamma(N+1)(\Gamma(n+1))^{2} \Gamma(-n)} \exp \left[-\left(k_{0} n(n+1)+k \pi^{2}\left(\frac{m^{2}}{b^{2}}+\frac{l^{2}}{c^{2}}\right)\right) t\right] \\
& \times P_{n}(x) \cos \frac{m \pi}{b} y \cos \frac{l \pi}{c} z
\end{aligned}
$$




$$
\times \aleph_{p_{i}+2, q_{i}+1, \tau_{i} ; R: W}^{0, \mathbf{n}+2: V}\left(\begin{array}{c|c}
p_{1} 2^{m_{1}^{\prime}+m_{1}^{\prime \prime}} & \left(-N: m_{1}^{\prime}, \cdots, m_{r}^{\prime}\right),\left(0: m_{1}^{\prime \prime}, \ldots, m_{r}^{\prime \prime}\right), A \\
\vdots & \vdots \\
p_{r} 2^{m_{r}^{\prime}+m_{r}^{\prime \prime}} & \left(-N-1: m_{1}^{\prime}+m_{1}^{\prime \prime}, \ldots, m_{r}^{\prime}+m_{r}^{\prime \prime}\right), B
\end{array}\right)
$$

where $P_{n}(x)$ is a Legendre's polynomial, under the same condition that (3.4) with $\alpha=\beta=0$.

\section{Aleph-Function of Two Variables}

If $r=2$, the multivariable Aleph-function reduces to Aleph-function of two variables defined by Sharma [13] (see also, [6]) and the general solution is

$$
\begin{aligned}
& v(x, y, z, t) \\
& =\frac{\mu \delta e^{-(\mu b+\delta c)} \Gamma(\alpha+1)}{b c 2^{\alpha+\beta-1}} \sum_{n, m, l, N=0}^{\infty} \frac{\left(1-(-)^{m}\right)\left(1-(-)^{l}\right)}{\left(\mu^{2}+m^{2} \pi^{2} / b^{2}\right)\left(\delta^{2}+l^{2} \pi^{2} / c^{2}\right)} \\
& \times \frac{n !(\alpha+\beta+2 n+1) \Gamma(\alpha+\beta+n+N+1) \Gamma(-n+N)}{N ! \Gamma(\alpha+N+1) \Gamma(\alpha+n+1) \Gamma(\beta+n+1) \Gamma(-n)} \\
& \times \exp \left[-\left(k_{0} n(n+\alpha+\beta+1)+k \pi^{2}\left(\frac{m^{2}}{b^{2}}+\frac{l^{2}}{c^{2}}\right)\right) t\right] P_{n}^{(\alpha, \beta)}(x) \cos \frac{m \pi}{b} y \cos \frac{l \pi}{c} z \\
& \times \aleph_{p_{i}+2, q_{i}+1, \tau_{i} ; R: W}^{0, \mathbf{n}+2: V}\left(\begin{array}{c|c}
p_{1} 2^{m_{1}^{\prime}+m_{1}^{\prime \prime}} & \left(-\alpha-N: m_{1}^{\prime}, m_{2}^{\prime}\right),\left(-\beta: m_{1}^{\prime \prime}, m_{2}^{\prime \prime}\right), A \\
\vdots & \vdots \\
p_{2} 2^{m_{2}^{\prime}+m_{2}^{\prime \prime}} & \left(-\alpha-\beta-N-1: m_{1}^{\prime}+m_{1}^{\prime \prime}, m_{2}^{\prime}+m_{2}^{\prime \prime}\right), B
\end{array}\right),
\end{aligned}
$$

under the same condition that (3.4) with $r=2$.

\section{I-Function of Two VARiables}

If $r=2$ and $\tau_{i}, \tau_{i^{\prime}}, \tau_{i^{\prime \prime}} \rightarrow 1$ the multivariable Aleph-function reduces to $I$-function of two variables defined by Sharma and Mishra [12] (see also, [11]) and the general solution is

$$
\begin{aligned}
& v(x, y, z, t) \\
& =\frac{\mu \delta e^{-(\mu b+\delta c)} \Gamma(\alpha+1)}{b c 2^{\alpha+\beta-1}} \sum_{n, m, l, N=0}^{\infty} \frac{\left(1-(-)^{m}\right)\left(1-(-)^{l}\right)}{\left(\mu^{2}+m^{2} \pi^{2} / b^{2}\right)\left(\delta^{2}+l^{2} \pi^{2} / c^{2}\right)} \\
& \times \frac{n !(\alpha+\beta+2 n+1) \Gamma(\alpha+\beta+n+N+1) \Gamma(-n+N)}{N ! \Gamma(\alpha+N+1) \Gamma(\alpha+n+1) \Gamma(\beta+n+1) \Gamma(-n)} \\
& \times \exp \left[-\left(k_{0} n(n+\alpha+\beta+1)+k \pi^{2}\left(\frac{m^{2}}{b^{2}}+\frac{l^{2}}{c^{2}}\right)\right) t\right] P_{n}^{(\alpha, \beta)}(x) \cos \frac{m \pi}{b} y \cos \frac{l \pi}{c} z \\
& \times I_{p_{i}+2, q_{i}+1, R: W}^{0, \mathbf{n}+2: V}\left(\begin{array}{c|c}
p_{1} 2^{m_{1}^{\prime}+m_{1}^{\prime \prime}} & \left(-\alpha-N: m_{1}^{\prime}, m_{2}^{\prime}\right),\left(-\beta: m_{1}^{\prime \prime}, m_{2}^{\prime \prime}\right), A \\
\vdots & \vdots \\
p_{2} 2^{m_{2}^{\prime}+m_{2}^{\prime \prime}} & \left(-\alpha-\beta-N-1: m_{1}^{\prime}+m_{1}^{\prime \prime}, m_{2}^{\prime}+m_{2}^{\prime \prime}\right), B
\end{array}\right)
\end{aligned}
$$


under the same condition that (3.4) with $r=2$ and $\tau_{i}, \tau_{i^{\prime}}, \tau_{i^{\prime \prime}} \rightarrow 1$.

\section{Concluding Remarks}

Specializing the parameters of the multivariable Aleph-function, we can obtain a large number of results involving various special functions of one and several variables useful in Mathematics analysis, Applied Mathematics, Physics and Mechanics. The result derived in this paper is of general character and may prove to be useful in several interesting situations appearing in the literature of sciences.

\section{REFERENCES}

[1] F. Ayant, An integral associated with the Aleph-functions of several variables, International Journal of Mathematics Trends and Technology 31 (2016), 142-154.

[2] F. Ayant and D. Kumar, A unified study of fourier series involving the Aleph-function and the Kampé de Fériet's function, International Journal of Mathematics Trends and Technology $\mathbf{3 5}$ (2016), 40-48.

[3] F. Ayant and D. Kumar, Generating relations and multivariable Aleph-function, Analysis 38 (2018), 137-143.

[4] H. Carslaw and J. Jaeger, Conduction of Heat Solids, Clarendon Press Oxford, Oxford, 1974.

[5] A. Erdélyi, Tables of Integrals Transforms, Vol. II, McGraw-Hill, New York, 1954.

[6] D. Kumar, Generalized fractional differintegral operators of the Aleph-function of two variables, Journal of Chemical, Biological and Physical Sciences, Section C 6 (2016), 1116-1131.

[7] D. Kumar, F. Ayant and D. Kumar, A new class of integrals involving generalized hypergeometric function and multivariable Aleph-function, Kragujevac J. Math. 44 (2020), 539-550.

[8] D. Kumar, J. Ram and J. Choi, Certain generalized integral formulas involving Chebyshev Hermite polynomials, generalized $M$-series and Aleph-function, and their application in heat conduction, Int. J. Math. Anal. 9 (2015), 1795-1803.

[9] I. Simões, A. Tadeu and N. Simões, Green's functions for heat conduction for unbounded and bounded rectangular spaces: time and frequency domain solutions, J. Appl. Math. 2016 (2016), $1-22$.

[10] Y. Prasad and R. Maurya, Application of Jacobi polynomial and multivariable H-function in heat conduction in non-homogeneous moving rectangular parallelepiped, Bulletin Mathematiques de la Societé des Sciences 24 (1980), 393-400.

[11] C. Sharma and S. Ahmad, On the multivariable I-function, Acta Ciencia Indica: Mathematics 20 (1994), 113-116.

[12] C. Sharma and P. Mishra, On the I-function of two variables and its properties, Acta Ciencia Indica: Mathematics 17 (1991), 667-672.

[13] K. Sharma, On the integral representation and applications of the generalized function of two variables, International Journal of Mathematical Engineering and Science 3 (2014), 1-13.

[14] H. Srivastava and R. Panda, Some expansion theorems and generating relations for the $\mathrm{H}$ function of several complex variables, Comment. Math. Univ. St. Pauli 24 (1975), 119-137.

[15] H. Srivastava and R. Panda, Some bilateral generating function for a class of generalized hypergeometric polynomials, J. Reine Angew. Math. 283/284 (1976), 265-274.

[16] N. Südland, J. Volkmann and D. Kumar, Applications to give an analytical solution to the Black Scholes equation, Integral Transforms Spec. Funct. 30 (2019), 205-230. 
${ }^{1}$ Department of Applied Sciences,

College of Agriculture, Sumerpur- Pali,

Agriculture University of Jodhpur, Jodhpur 342304, India

Email address: dinesh_dino03@yahoo.com

${ }^{2}$ Collége Jean L'Herminier,

Allée des Nymphéas, 83500 La Seyne-Sur-Mer, France

${ }^{3}$ Six-Fours-les-Plages-83140, Department of VAR, France

Email address: fredericayant@gmail.com 